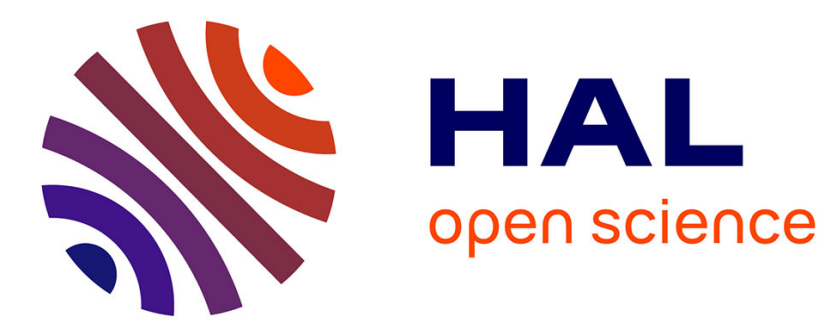

\title{
On the influence of dynamical effects on reactivity descriptors
}

Guillaume Hoffmann, Vincent Tognetti, Laurent Joubert

\section{To cite this version:}

Guillaume Hoffmann, Vincent Tognetti, Laurent Joubert. On the influence of dynamical effects on reactivity descriptors. Chemical Physics Letters, 2019, 724, pp.24-28. 10.1016/j.cplett.2019.03.040 . hal-02330543

\section{HAL Id: hal-02330543}

\section{https://hal-normandie-univ.archives-ouvertes.fr/hal-02330543}

Submitted on 22 Oct 2021

HAL is a multi-disciplinary open access archive for the deposit and dissemination of scientific research documents, whether they are published or not. The documents may come from teaching and research institutions in France or abroad, or from public or private research centers.
L'archive ouverte pluridisciplinaire HAL, est destinée au dépôt et à la diffusion de documents scientifiques de niveau recherche, publiés ou non, émanant des établissements d'enseignement et de recherche français ou étrangers, des laboratoires publics ou privés.

\section{다)(1) $(5$}

Distributed under a Creative Commons Attribution - NonCommerciall 4.0 International 


\title{
On the influence of dynamical effects on reactivity descriptors
}

Guillaume HOFFMANN, Vincent TOGNETTI*, Laurent JOUBERT*

Normandy Univ., COBRA UMR 6014 \& FR 3038, Université de Rouen, INSA Rouen, CNRS, 1 rue Tesniére 76821 Mont St Aignan. Cedex, France

\begin{abstract}
In this paper, we investigate the role of structural dynamical effects on reactivity properties of selected Michael acceptors. To this aim, quantum molecular dynamics simulations were performed. Conceptual density functional theory descriptors were then evaluated on selected snapshots, providing statistical indicators suitable to assess the discrepancies between static and dynamical approaches. The implications of these results for building a predictive model correlated to Mayr's electrophilicity index are then discussed, paving the way towards a more realistic account of experimental results.
\end{abstract}

Keywords: Michael acceptors, Electrophilicity, Conceptual DFT, Reactivity indices, Molecular Dynamics, Mayr's scale

Corresponding authors: vincent.tognetti@univ-rouen.fr, laurent.joubert@univ-rouen.fr 


\section{Introduction}

Conceptual density functional theory (CDFT) [1-4] and the respective chemical reactivity indices that it generates were shown successful in the prediction and characterization of empirical and theoretical concepts, such as electrophilicity [5-9], nucleophilicity [10-12], gas-phase basicities [13], Woodward-Hoffmann rule's [14], $\sigma$-holes [15,16], trans-effect [17,18], and covering all research fields ranging from organic to inorganic chemistries. In particular, they provide an efficient prediction of the feasibility of a chemical reaction based only on the reactants physicochemical properties. In the case of electrophilicity and nucleophilicity, these theoretical descriptors can be confronted to the corresponding experimental parameters defining the popular Mayr's scales [19-21].

Noteworthy, these scales are widely used by experts in organic synthesis for a quick assessment of the outcome of a reaction between known compounds. Indeed, a reliable rule of thumb is that the reaction of a nucleophile and an electrophile will be quantitative if the sum of their Mayr's parameters is higher than -5. Currently, there are 1456 available experimental parameters for reagents such as diverse as carbocations, aldehydes, ketones, imines, alkenes, ylides, aliphatic amines..., thus covering most of the usual arsenal used in organic synthesis.

It currently remains a theoretical challenge to predict these experimental parameters (based on kinetic measurements) for any type of molecule. Certainly, such a predicting tool would be highly valuable for organic chemists dealing with compounds for which experimental parameters have not been yet determined, and even for biologists, for example in the assessment of toxicity of molecules [22]. Besides this predictive power, such a model could also convey chemical rationalization, which could help improving the current reagents by a well-targeted in silico strategy. 
In a preceding study [23], we developed a model able of categorizing the electrophilicity reactivity of $\alpha$ - $\beta$-unsaturated compounds, which epitomize Michael acceptors, a family of chemical reagents instrumental in the formation of carbon-carbon bonds and ubiquituous in organic synthesis. This model, as almost all of related works (see also reference 24), was based on a static approach of chemical reactivity, and failed at providing a quantitative model with respect to experimental data, a fact also underlined by Mayr in recent studies devoted to Michael acceptors [25,26] and later extended to other reagents like ketones [27].

One may thus wonder whether this failure might be ascribable to the neglect of dynamical features. Indeed, due to thermal agitation, the reactants inside a beaker are generally not frozen in their most stable conformation. This structural breathing may in some cases lead to explore geometries that can significantly differ from the optimized ones, and which would accordingly exhibit significantly different reactivity behaviors.

From the best of our knowledge, the calculation of chemical reactivity descriptors extracted from dynamical trajectories is extremely scarce, probably because static approaches often revealed sufficient to correctly predict experimental results, as exemplified by the benzhylidrium carbocations family for which many high linear correlations were reported and that has served as a workhorse for electrophicility studies for a long time.

As a matter of fact, the predominant focus on rigid or hindered species of such type could account for the prevalence of the static theoretical approach that is, furthermore, much less timeconsuming from a computational perspective. Nevertheless, we want to point out that the dynamical behavior of chemical reactivity indices was seminally investigated by Liu [28] in gas phase for few hydrated clustered cations or anions (like $\mathrm{Ca}^{2+}\left(\mathrm{H}_{2} \mathrm{O}\right)_{15}$ or $\left.\mathrm{Cl}^{-}\left(\mathrm{H}_{2} \mathrm{O}\right)_{30}\right)$, but with the main focus not being organic chemical reactivity. Another important study is that presented as a 
poster by Oller and Vöhringer-Martinez during the QUITEL 2018 conference held in Santiago (Chile), which reports a methodology based on molecular dynamics to define Boltzmann weighted reactivity descriptors [29].

In the current work, molecular dynamics simulations in solution on selected Michael acceptors were performed at a semi-empirical quantum chemical level of theory in order to follow the evolution over time of some of the most relevant reactivity descriptors considered in our previous paper [23], and to assess to what extent they differ from their static properties and may afford hints on how to improve reactivity prediction by means of conceptual DFT.

\section{Computational Details}

All density functional theory calculations were performed using the Gaussian 09 package [30]. Consistently with our previous study [23], molecular geometries were first optimized using the meta-hybrid M06-2X exchange-correlation functional [31] in conjunction with the aug-cc-pVTZ basis set, with recommended 'ultrafine' integration grid. Solvent effects (dimethylsulfoxide, DMSO) were taken into account by a polarizable continuum model (in the last implementation of the integral equation formalism (IEFPCM) [32]) for all computations (static and dynamical approaches).

These optimized structures were then used to start unbiased molecular dynamics simulations at the quantum chemical semi-empirical PM6 level of theory [33] (within IEFPCM), which allows long simulations at a low computational cost, in the NVT canonical ensemble. The time step for propagation was set to 1 fs. The PM6 wavefunction was converged at any step (Born- 
Oppenheimer-type dynamics). The total simulation time was equal to $100 \mathrm{ps}$, ensuring representative sampling. Temperature $(\mathrm{T}=298.15 \mathrm{~K})$ was controlled by velocity scaling.

Structures were then extracted every 1 ps. The 20 first ones were considered to be part of the equilibration process, while the 80 following ones were considered for data production. Indeed, as shown in Graph S1 in the supplementary information file, temperature was found to be properly converged after 20 ps for every simulation. Subsequent single point calculations at the M06-2X/aug-cc-pVTZ level of theory were carried out to evaluate the chosen conceptual DFT reactivity descriptors.

These descriptors can be classified into two main classes: molecular (also known as "global") and atomic ones. Those belonging to the first category are here: three avatars of the electronic chemical potential $\left(\mu, \mu^{+}, \mu\right)$, the molecular hardness $\eta$, softness $S$, and hyperhardness $\gamma$ [34], the electrophilicity index $\omega[35,36]$, and the electroaccepting power $\omega^{+}$. Atomic descriptors considered here are: condensed electrophilic and nucleophilic Fukui functions $f^{+}, f^{-}$, and the dual descriptor $f^{(2)}[37,38]$, supplemented by grand-canonical extensions $\left(\mu^{+} f^{+}, \mu f^{-}, \omega f^{+}, S f^{(2)}, \omega f^{(2)}\right.$, $\left.s^{(2)}, s^{+}=S f^{+}, s^{-}=S f^{-}\right)$. We refer the interested reader to our previous paper for the corresponding mathematical definitions [23].

Besides, two main computational approaches can be implemented to evaluate these descriptors: either finite difference linearization (FDL) or the Koopmans-like frozen molecular orbital (FMO) scheme, which are not equivalent [39]. For each descriptor, both were here considered. Atomic condensation was performed within the framework of Bader's atoms-in-molecules theory (QTAIM)[40,41] using Todd Keith's AIMAll software [42]. 
It is important to notice that two carbon atoms denoted $\mathrm{C} \alpha$ and $\mathrm{C} \beta$ (see Figure 1) may be involved in the carbon-carbon bond formation process during a Michael addition, so that condensation of the two atomic corresponding basins was performed. In ref. 23, we also defined the total electrophilicity of the $\mathrm{C}_{\alpha}=\mathrm{C} \beta$ double bond, $\mathrm{f}^{+}\left(C_{\alpha \beta}\right)$, by simply summing the electrophilicity of each atom, $\mathrm{f}^{+}\left(C_{\alpha}\right)$ and $\mathrm{f}^{+}\left(C_{\beta}\right)$.

In total, for each molecule, 9 global descriptors and 22 condensed ones were evaluated at both the FDL and FMO theory levels at each selected point along the dynamical trajectory.

Atomic units (a.u.) are used throughout the paper unless explicitly otherwise stated. Numerical values corresponding to the statistical analysis of all descriptors are gathered in the supplementary information file (Tables S1-S80). Experimental values were extracted from Mayr's online database [43].

\section{Results and discussion}

In order to investigate dynamical effects on chemical reactivity indices, we selected 11 molecules (represented in Figure 1) out of our previous dataset [23] that consisted of 35 Michael acceptors. Let us recall that we divided it into three subgroups: "good" Michael acceptors, "medium", and "low" ones. In order to be as representative as possible, we thus selected in the current paper molecules stemming from each of these three representative families. Note that two molecules of these sets are symmetric (compounds $\mathbf{1}$ and $\mathbf{8}$ ) at their energy minimum, a property that will not be kept during the molecular dynamics at a given time. However, the time-averaged properties 
for the two carbons that are equivalent in the optimized structures were found, as expected, almost equal at the end of the simulation.

In summary, this new working subset covers a range of experimental electrophilicity values from -11.31 to -23.54 , which is that of the known experimental one for Michael acceptors, with values well distributed along it. To be more comprehensive, let us recall that the nucleophilic addition on a Michael acceptor that features conjugated multiple bonds (for instance $\mathrm{C}=\mathrm{C}$ and $\mathrm{C}=\mathrm{O}$ as in 11, or $\mathrm{C}=\mathrm{C}$ and $\mathrm{C} \equiv \mathrm{N}$ as in 7) affords the formation of a bond between the nucleophile (often a carbanion) and carbone $\mathrm{C} \beta$ of the acceptor (see Figure 1 for atom labeling).

As a first example for the analysis of variation of descriptors along a trajectory, we now scrutinize the electrophilicity index (calculated using FDL) for N,N-dimethylprop-2-enamide (molecule 11). Its values during the simulation are displayed in the left part of Figure 2. The static value was found equal to 0.061 a.u. It can be compared to the average value (calculated on the last 80 ps): 0.057 a.u., and to the median: 0.054 a.u. The obtained values spanned between 0.046 a.u. ( $\min )$ and 0.077 a.u. ( $\max )$, and the standard deviation is 0.009 a.u., so that the coefficient of variation is equal to $16 \%$. This last value is not at all negligible, showing that even for a rigid molecule as 11, dynamical effects can be of importance. Interestingly, the average and median values suggest that the molecule is actually less reactive than expected from the static approach.

One may wonder if this is a general result for the FDL electrophilicity index. It is actually the case for compound $\mathbf{1 0}$ (static value: 0.091, average: 0.075 ) and $\mathbf{3}$ (static: 0.122, average: 0.115 ). Note that these two molecules show different behaviors: important discrepancies between the two approaches for 10, small ones for 3. Conversely, the trend was found opposite for $\mathbf{2}$ (static value: 
0.079, average: 0.094 ) and $\mathbf{1}$ (static: 0.120 , average: 0.124 ). It is also instructive to compare 10 and 2: the electrophilicity index is higher from the static point view for $\mathbf{1 0}$, while it is higher in $\mathbf{2}$ from the average side.

It is also instructive to compare values for FMO electrophilicity index values. At the static level, the values are almost identical for compounds $\mathbf{2}$ and $\mathbf{1 0}$ ( 0.060 and 0.062 , respectively), while the average values considerably differ: 0.069 (molecule 2) with respect to $0.052(\mathbf{1 0})$. This is in line with the fact that Mayr's electrophilicity is significantly higher for $\mathbf{2}$ than for $\mathbf{1 0}(-12.1$ vs. -23.0$)$. All these preliminary results prove that considering a dynamical approach can invert reactivity orders, and possibly significantly alter the reactivity hierarchy.

As a second example, we now have a look at an atomic descriptor, and more specifically at the dual descriptor condensed on carbon $\mathrm{C} \beta$ for compound 10, namely (E)-4-phenylbut-3-en-2-one. Let us recall that the dual descriptor allows for predicting if an atom is mainly electrophilic (positive condensed value) or nucleophilic (negative condensed value). The time evolution of the dual descriptor $f_{F D L}^{(2)}\left(C_{\beta}\right)$ is represented in the right part of Figure 2. It appears that the static, median and average values are all positive, but that the two first quantities differ a lot, since these three values were found equal to $0.012,0.057,0.055$, respectively.

Another salient feature is that there are several sign changes along the trajectory, the most important one occurring around $t=55 \mathrm{ps}$. This means that the carbon atom then changes its reactivity from electrophilic to nucleophilic. One then would have expected the average value to be lower than the static one due to these negative contributions. This is actually not the case because they are largely compensated by important positive values (see for instance the positive 
peak before $t=80 \mathrm{ps}$ ). As a consequence, this compound exhibits a very high coefficient of variation value since it amounts to $84 \%$.

All of these statistical quantities were evaluated for all molecules. By doing so, the average, minimal, maximal, and median values become new reactivity descriptors that can be used to predict Mayr's experimental electrophilicity, denoted $E_{\text {Mayr }}$, for the subset of 11 Michael acceptors. In fact, for a given descriptor, the dynamical values are generally not highly correlated to the static ones, as shown in Tables S81-S88 in the supplementary information file that gather selected correlation matrices. For instance, the coefficient of determination between the average and the static $f_{F D L}^{(2)}\left(C_{\beta}\right)$ values was found equal to 0.66 . This implies that these dynamical descriptors are true new descriptors.

A simple way for comparing their prediction ability with static ones is to compare their coefficient of determination (for monolinear regressions) towards Mayr's experimental electrophilicity. To this aim, Figure 3 shows the $R_{\text {median }}^{2}$ values from dynamical simulations with respect to the $R_{\text {static }}^{2}$ ones for all tested descriptors.

This analysis showed that for some descriptors, dynamics effects do not significantly modify the correlation to the experimental value. This is for instance the case for $\mu_{F D L}, S_{F D L}$ and $\gamma_{F D L}$. Indeed, for the electronic chemical potential, we found $R_{\text {median }}^{2}=0.74$ and $R_{\text {static }}^{2}=0.72$. Likewise, all descriptors with very low $R^{2}$ values (lower than 0.2 ) in the static case also exhibit low $R^{2}$ dynamical values.

On the contrary, we obtained important differences for some other reactivity descriptors. This is notably the case for $\omega_{F D L}, \mu_{F D L}^{+}$and $\omega_{F D L}^{+}$, the corresponding points being circled in Figure 3. For the electrophilicity index, we got $R_{\text {median }}^{2}=0.71$ and $R_{\text {static }}^{2}=0.42$. These results indicate that 
some descriptors that were not highly correlated to experimental electrophilicity in the static approach can be now considered as promising tools for the global prediction. More generally, it was found that $R_{\text {median }}^{2}$ values are generally higher than static ones, corresponding to points located in the blue area in Figure 3. Similar conclusions hold for the other statistical descriptors, in particular the time-averaged values.

More precisely, the highest $R^{2}$ values obtained using all static and dynamical values in monolinear regression procedure was found with the median value of $\omega_{F M O}$, for which $R^{2}$ is equal to 0.77 . If only static descriptors were retained, this highest $R^{2}$ value dropped down to 0.55 . Henceforth, there is a little improvement, but it can be considered not sufficient to achieve quantitative correlation. In fact, as discussed in our previous paper [23], at least two descriptors should be used for correlation when the dataset is composed of very different molecules in order to grasp the various possible reactivity behaviors (charge or orbital control, role of the two carbons of the double bond in the chemical process...).

We then performed bilinear regression analysis. In order to only deal with consistent combinations, only FDL (resp. FMO) descriptors were paired with another FDL (resp. FMO) one. For the very same reasons, we only coupled statistical descriptors of the same kind: for instance, an average value with another average value, excluding mixed combinations (an average value with a maximal one, for example). Ten descriptor subsets were thus subjected to bilinear regressions, defined by FMO static, FDL static, FMO average, FDL average, FMO median, FDL median, FMO maximal, FDL maximal, FMO minimal, and finally FMO maximal values. Table 1 collects the obtained models with the highest $R^{2}$ values.

When using only static descriptors, the best models showed $R^{2}$ of about 0.8 . Noteworthy, such values indicate a prediction capacity that is similar to the one we reported for the full dataset of 
35 molecules of our previous paper [23] (this likeness also confirms that the current subset can be considered as statistically representative of the whole one). The model with the highest $R^{2}$ value was the following one:

$$
E_{\text {Mayr }}=-54.39+344.92 \mu_{F M O, \text { static }}^{+}+821.34 \omega_{F M O, \text { static }} .
$$

It is worth noticing that it involves only global descriptors, which is an advantage since it is independent of any atomic partition and since its evaluation is more straightforward than condensed values. Nevertheless, the $R^{2}$ value remains rather low. One can then wonder if it can be improved using statistical global descriptors. This is actually the case, involving other descriptors:

$$
E_{\text {Mayr }}=-42.99-169.60 \mu_{F D L, \text { average }}-55.35 \gamma_{F D L, \text { average }},
$$

with $R^{2}=0.86$. Substantial predictivity enhancement can thus be achieved only incorporating condensed descriptors from the dynamical approach. The most promising models were actually obtained using the medians $\left(\mathrm{R}^{2}=0.93\right)$ according to:

$$
E_{\text {Mayr }}=-14.04-42.46 s_{F D L, \text { median }}^{+}\left(C_{\alpha}\right)+1836.84 \omega f_{F D L, \text { median }}^{+}\left(C_{\alpha}\right) .
$$

and with the average values $\left(R^{2}=0.97\right)$ following:

$$
E_{\text {Mayr }}=-14.58-44.84 s_{F D L, \text { average }}^{+}\left(C_{\alpha}\right)+1996.99 \omega f_{F D \text {, average }}^{+}\left(C_{\alpha}\right) .
$$

Note that here $\omega f_{\text {median }}^{+}$refers to the median of the whole $\omega\left(t_{i}\right) f^{+}\left(t_{i}\right)$ value distribution along the trajectory (at times $t_{i}$ ). It is not equal to the average value of $\omega$ multiplied by the average of $f^{+}$values.

Our last model (represented in Figure 4) is thus particularly appealing since its mean absolute deviation with respect to Mayr's experimental values is equal to 0.6 relative units, to be 
compared to 1.6 that was obtained with equation 1 from the static approach.. A striking feature of equations 3 and 4 is that they involve properties related to carbon $\alpha$ and not to carbon $\beta$, while the bond between nucleophile and electrophile occurs on carbon $\beta$. This is an important point we already discussed in our previous paper [23]. Actually, if we restrained the bilinear regression to $\mathrm{C} \beta$ descriptors, the best model corresponds to:

$$
E_{\text {Mayr }}=-32.37-14,59 f_{F D L, \text { maximal }}^{(2)}\left(C_{\beta}\right)+1053.59 \omega^{+} f_{F D L, \text { maximal }}^{+}\left(C_{\beta}\right),
$$

with $R^{2}=0.84$, featuring an obvious worsening of the model.

Obviously, bilinear models are relevant if they combine descriptors that are not highly correlated. We thus computed the intercorrelation matrix gathering the $R^{2}$ values between each pair of descriptors entering equations 1-5. It corresponds to Table S88 in the supplementary information file. It immediately appears that such descriptors cannot be considered correlated, so that those involved in the bilinear models are really complementary.

Lastly, in order to roughly assess the reliability of such models, we considered a molecule that was not included in the fitting set, namely fumaronitrile. Its Mayr's electrophilicity is equal to 15.71, filling the missing gap between compounds $4\left(E_{\text {Mayr }}=-16.76\right)$ and $\mathbf{3}\left(E_{\text {Mayr }}=-13.85\right)$. The static values for $S_{F D L}^{+}\left(C_{\alpha}\right)$ and $\omega f_{F D L}^{+}$were found to be equal to 0.527 and 0.013 , respectively, to be compared to the corresponding time-averaged ones: 0.564 and 0.012 . The use of equation 4 then predicted an electrophilicity equal to -15.31 . The difference between this last value and the experimental one is equal to 0.4 , and it is thus lower than the mean absolute deviation calculated on the 11 molecules used to build the model. This new result supports once more the promising predictive power of dynamical reactivity descriptors. 
Finally, it should also be noticed that, unfortunately, such models are far from being easily interpreted since it involves a competition between $S_{F D L \text {,average }}^{+}\left(C_{\alpha}\right)$ and $\omega f_{F D L \text {,average }}^{+}$that both enter equation 4 with opposite signs. There are in fact cases where the sum of these two contributions is positive and other ones for which it reveals negative. We believe that such facts could not simply be accounted for without resorting to a detailed mechanistic study of these reactions, which is outside the scope of this paper and that we will report in due course.

\section{Conclusions}

In this letter, we have shown that dynamical effects can trigger dramatic changes of reactivity descriptor values with respect to those obtained from a static approach. We also gave evidence that average and median statistical data gathered along a trajectory allows for a much better prediction of Mayr's electrophilicity parameters. It also paves the way toward the definition of temperature-dependent reactivity indices (by choosing the appropriate thermostat temperature for

the molecular dynamics simulations), and therefore toward more realistic explanation of experimental data. Besides, such a protocol is general and could be applied to other families than Michael acceptors.

\section{Acknowledgements}

The authors would like to gratefully acknowledge the LABEX SynOrg and the Normandy Region for funding and support, and the CRIANN high-performance computing facility. Part of this project was supported by the RIN AGAC project. 


\section{Supplementary information}

Tables with statistical analysis of all descriptors and graphs showing temperature convergence. 


\section{References}

[1] R.G. Parr, W. Yang, Annu. Rev. Phys. Chem. 46 (1995) 701.

[2] H. Chermette, J. Comput. Chem. 20 (1999) 129.

[3] P. Geerlings, F. De Proft, W. Langenaeker, Chem. Rev. 103 (2003) 1793.

[4] F. De Proft, P. Geerlings, Chem. Rev. 101 (2001) 1451.

[5] L.R. Domingo, P. Pérez, R. Contreras, Tetrahedron 60 (2004) 6585.

[6] A. Aizman, R. Contreras, P. Pérez, Tetrahedron 61 (2005) 889.

[7] V. Tognetti, C. Morell, L. Joubert, Chem. Phys. Lett. 635 (2015) 111.

[8] L.G. Zhuo, W. Liao, Z.X. Yu, Asian J. Org. Chem. 1 (2012) 336

[9] X.Y. Zhou, C.Y. Rong, T. Lu, S. Liu, Acta Phys. Chim. Sin. 30 (2014) 2055.

[10] S. Liu, C. Rong, T. Lu, J. Phys. Chem. A. 118 (2014) 3698.

[11] C. Wang, Y. Fu, Q.X. Guo, S. Liu, Chem. Eur. J. 16 (2010) 2586.

[12] R. Contreras, J. Andres, V.S. Safont, P. Campodonico, J. G. Santos, J. Phys. Chem. 107 (2003) 5588.

[13] S.K. Rajak, N. Islam, D.C. Ghosh, J. Quantum Inf. Sci. 1 (2011) 87.

[14] P. Geerlings, P.W. Ayers, A. Toro-Labbé, P.K. Chattaraj, F. De Proft, Acc. Chem. Res. 45 (2012) 683.

[15] V. Tognetti, C. Morell, L. Joubert, J. Comput. Chem. 36 (2015) 649.

[16] N. Galland, G. Montavon, J-Y. Le Questel, J. Graton, New J. Chem. 42 (2018) 10510.

[17] F. Guégan, V. Tognetti, L. Joubert, H. Chermette, D. Luneau, C. Morell Phys. Chem. Chem. Phys. 18 (2016) 982.

[18] V. Tognetti, F. Guégan, D. Luneau, H. Chermette, C. Morell, L. Joubert, Theor. Chem. Acc. 136 (2017) 85.

[19] H. Mayr, M. Patz, Angew. Chem. Int. Ed. 33 (1994) 938.

[20] R. Lucius, R. Loos, H. Mayr, Angew. Chem. Int. Ed. 41 (2002) 91.

[21] H. Mayr, Angew. Chem. Int. Ed. 50 (2011) 3612.

[22] J.A.H. Schwobel, Y.K. Koleva, S.J. Enoch, F. Bajot, M. Hewitt, J.C. Madden, D.W. Roberts, T.W. Schultz, Chem. Rev. 111 (2011) 2562.

[23] G. Hoffmann, V. Tognetti, L. Joubert, J. Mol. Model. 24 (2018) 281.

[24] J. Oller, P. Pérez, P.W. Ayers, E. Vöhringer-Martinez, Int. J. Quantum Chem. 118 (2018) e25706. 
[25] D.S. Allgäuer, H. Jangra, H. Asahara, Z. Li, Q. Chen, H. Zipse, A.R. Ofial, H. Mayr, J. Am. Chem. Soc. 139 (2017) 13318.

[26] H. Jangra, Q. Chen, E. Fuks, I. Zenz, P. Mayer, A.R. Ofial, H. Zipse, H. Mayr, J. Am. Chem. Soc. 140 (2018) 16758.

[27] Z. Li, H. Jangra, Q. Chen, P. Mayer, A.R. Ofial, H. Zipse, H. Mayr, J. Am. Chem. Soc. $140(2018) 5500$.

[28] S. Liu, J. Chem. Sci. 117 (2005) 477.

[29] J. Oller, E. Vöhringer-Martinez, "Boltzmann weighted local reactivity descriptors of $\alpha, \beta$ unsaturated systems by molecular dynamics simulations", poster presentation in the XLIV International Congress of Theoretical Chemists of Latin Expression, Santiago, October $8^{\text {th }}-12^{\text {th }}$ 2018.

[30] M. J. Frisch, et al. Gaussian 09 Revision D.01, Gaussian Inc., Wallingford, CT, 2013.

[31] Y. Zhao , D. G. Truhlar, Theor. Chem. Acc. 120 (2008) 215.

[32] E. Cancès, B. Mennucci, J. Tomasi, J. Chem. Phys. 107 (1997) 3032.

[33] J.J.P. Stewart, J. Mol. Model. 13 (2007) 1173.

[34] C. Morell, A. Grand, A. Toro-Labbé, H. Chermette, J. Mol. Model. 19 (2013) 2893.

[35] R.G. Parr, L. von Szentpály, S. Liu, J. Am. Chem. Soc. 121 (1999) 1922.

[36] PK. Chattaraj, U. Sarkar, D.R. Roy, Chem. Rev. 106 (2006) 2065.

[37] C. Morell, A. Grand, A. Toro-Labbé, J. Phys. Chem. A 109 (2005) 205.

[38] C. Morell, A. Grand, A. Toro-Labbé, Chem. Phys. Lett. 425 (2006) 342.

[39] F. Zielinski, V. Tognetti, L. Joubert, Chem. Phys. Lett. 527 (2012) 67.

[40] R.F.W. Bader, Atoms in Molecules: A Quantum Theory, Oxford University Press, Oxford, U.K., 1990. [istep?

[41] P.L.A. Popelier, Atoms in Molecules An Introduction, Pearson Education, Harlow, 2000.

[42] T.A. Keith, AIMAll (Version 17.11.14), aim.tkgristmill.com, 2017.

[43] https://www.cup.lmu.de/oc/mayr/reaktionsdatenbank2/. 


\section{Figure captions}

Figure 1. View of the studied Michael acceptors. EWG stands for electron-withdrawing group. Numbers refer to experimental electrophilicities as measured by the Mayr's group (relative units).

Figure 2. Time (in ps) evolution of the electrophilicity index for molecule $\mathbf{1 1}$ (left part, atomic units) and of $\mathrm{C} \beta$ condensed dual descriptor for molecule $\mathbf{1 0}$ (right part, atomic units), obtained by single-point calculations at the M06-2X/aug-cc-pVTZ level of theory on snapshots from PM6 NVT molecular dynamics simulation.

Figure 3. Coefficients of determination $\left(R_{\text {median }}^{2}\right)$ for monolinear regressions using median values of each reactivity descriptor with respect to coefficients of determination $\left(R_{\text {static }}^{2}\right)$ using static values of the same descriptors.

Figure 4. Best bilinear model for the prediction of Mayr's electrophilicity scale (relative units) according to equation 4 . The straight line represents perfect agreement with experimental data. 


\section{$\beta \overbrace{\mathrm{EWG}}^{\alpha}$}

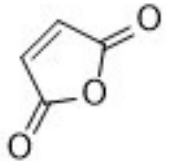

1

$-11.31$

$\mathrm{CN}$<smiles>CCOC/C=C\C(=O)OCC</smiles>

7

$-19.05$
$\widehat{\mathrm{SO}_{2} \mathrm{~F}}$

2

$-12.09$
$\mathrm{C}(\mathrm{O}) \mathrm{Me}$

4

$-13.85$

$-16.76$
$\mathrm{SO}_{2} \mathrm{Ph}$

5 6

$-18.84$
$\mathrm{CO}_{2} \mathrm{Me}$<smiles>CC(=O)C=Cc1ccccc1</smiles>

10

11

9

$-23.54$ 


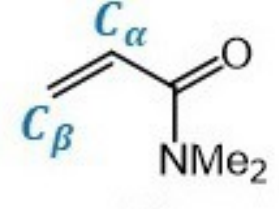

11

$$
f_{F D L}^{(2)}\left(C_{\beta}\right)(a . u .)
$$

0.20

10
0.080

0.075

0.070

0.065

0.060

0.055

0.050

0.045

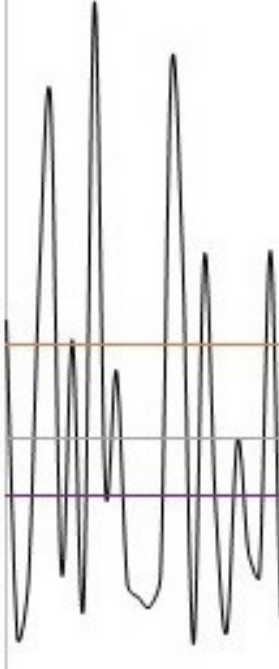

No

40

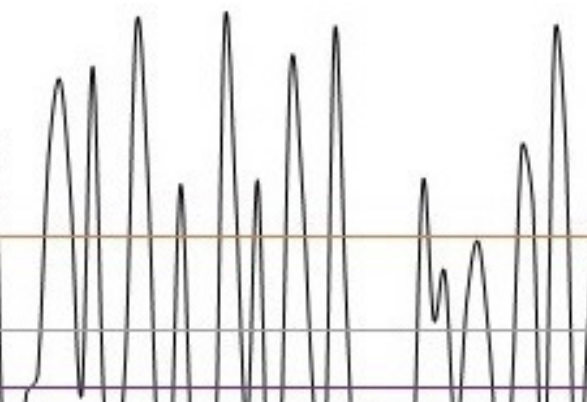

隌

60
0.15

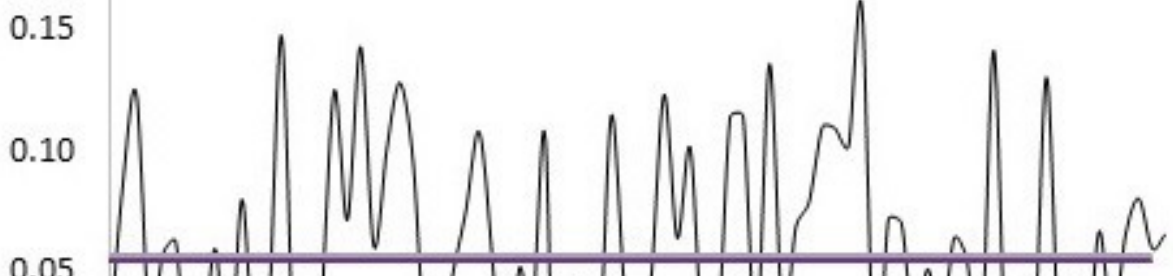

0.05

0.00

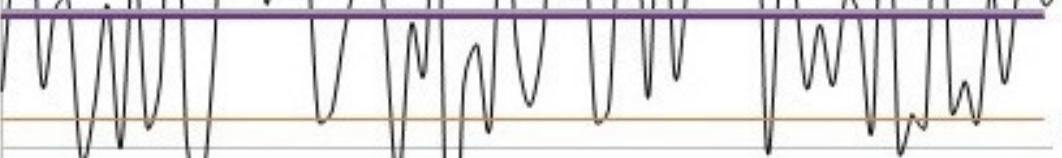

20
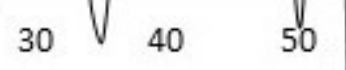

60

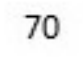

80

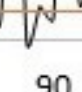

$t(p s)$

$-0.05$

$-0.10$

$t$ (ps)

- Static

-Average

—Median 


\section{$R_{\text {median }}^{2}$}

0.90

Better correlation using dynamical simulation

0.80

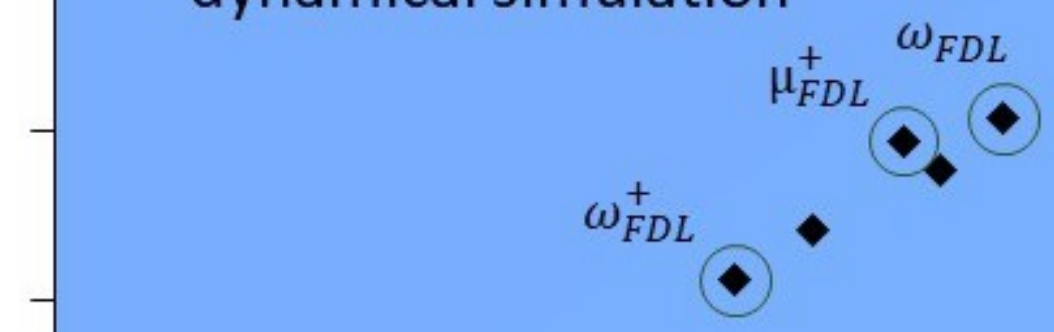

0.70

0.60

0.50

0.40

0.30

0.20

0.10
0.00

0.10
0.00

0.00

0.10

0.20

0.30

0.40

0.50

0.60

0.70

0.80

$R_{\text {static }}^{2}$ static approach

Better correlation using 
$E_{\text {Mayr }}$

$-5$

$$
R^{2}=0,97
$$

$-10$

$-15$

$-20$

$-25$

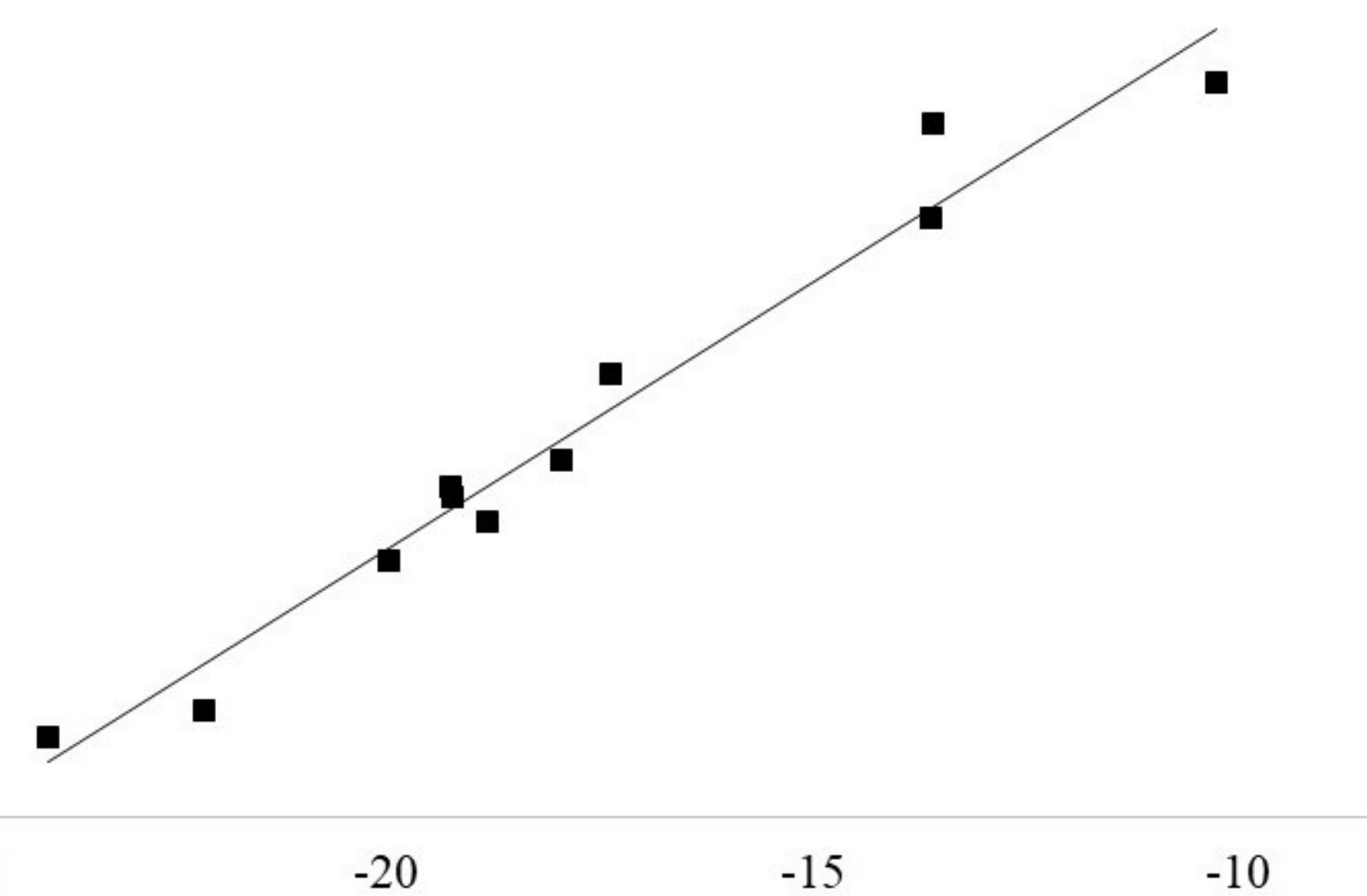

$E_{\text {model }}^{(2)}$

$\begin{array}{ll}-25 & -20\end{array}$

$-15$

$-10$

$-5$ 
TABLE 1. Bilinear regressions toward Mayr's electrophilicity scale with highest coefficient of determination values, depending on the nature of the used descriptors.

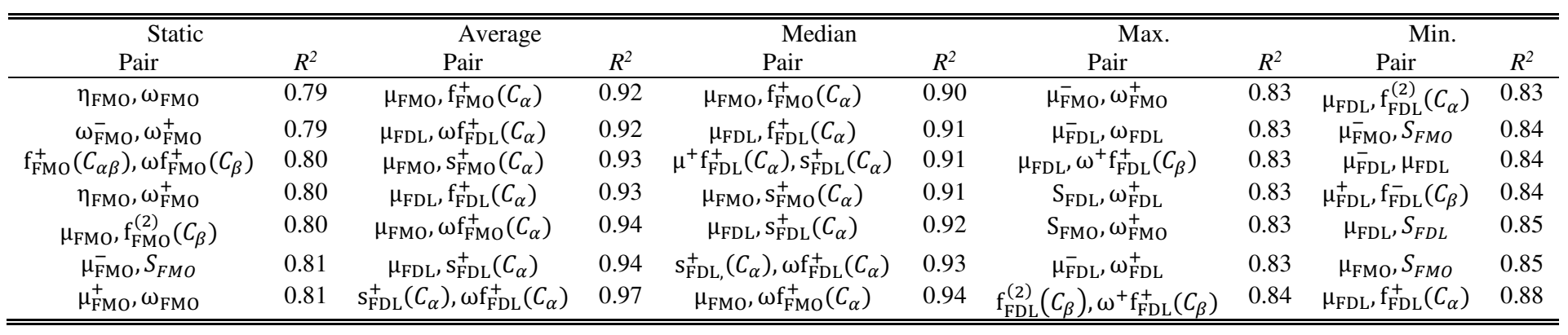


Michael Acceptors

Chemical Descriptors
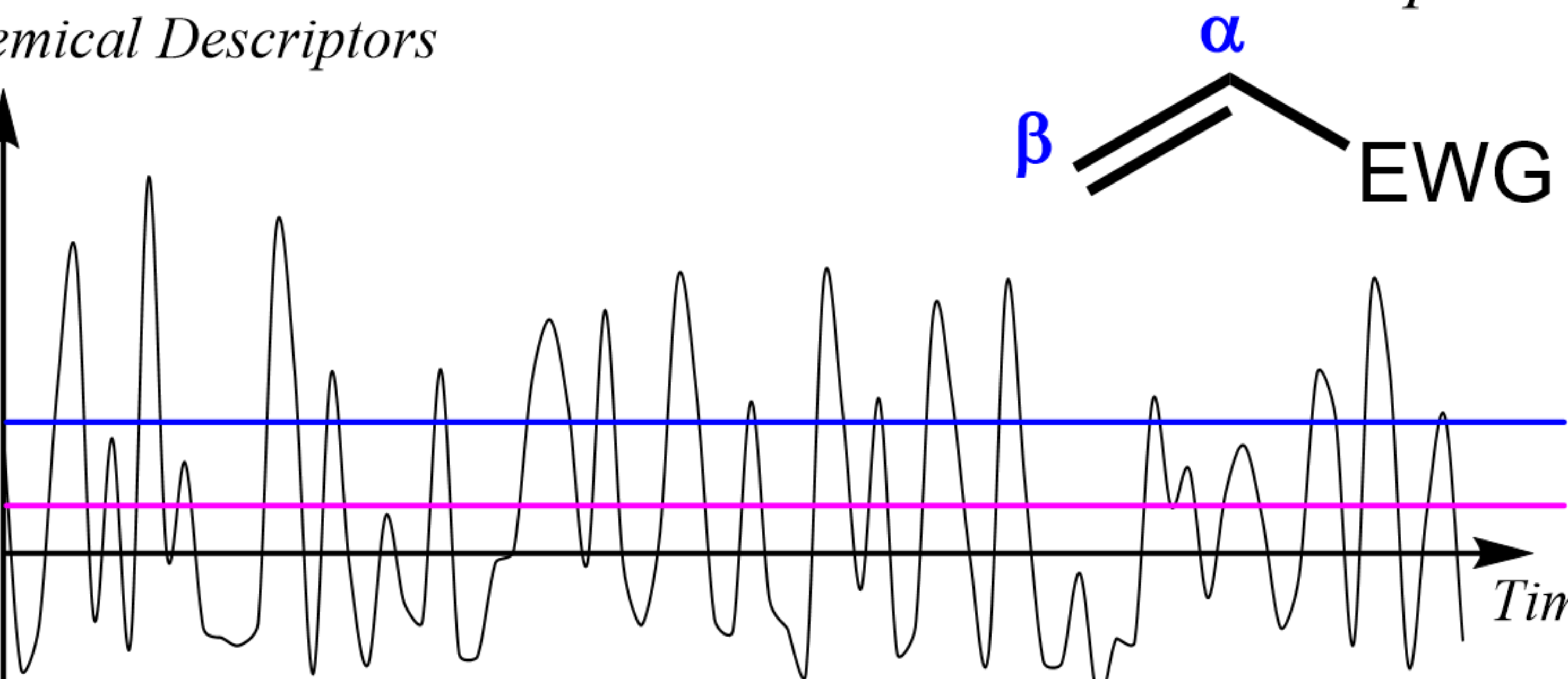

Towards a better prediction of Mayr's Electrophilicity 